\title{
Self-consistent simulation of GaAs/InGaAs/AIGaAs heterostructures photoluminescence spectra and its application to pHEMT structures diagnostics
}

\author{
(C) M.S. Mironova ${ }^{1}$, V.I. Zubkov ${ }^{1}$, A.L. Dudin ${ }^{2}$, G.F. Glinskii ${ }^{1}$ \\ ${ }^{1}$ St. Petersburg Electrotechnical University „LETI“, \\ 197376 St. Petersburg, Russia \\ 2 JSC „Svetlana-Rost“, \\ 194156 St. Petersburg, Russia \\ E-mail: mironova.m.s@gmail.com
}

We performed numerical self-consistent solution of Schrödinger and Poisson equations for $\mathrm{GaAs} / \mathrm{InGaAs} / \mathrm{AlGaAs}$ pHEMT structures with quantum well. Based on the results we calculated optical transition matrix elements and photoluminescence spectra of such structures with the same design and different parameters (such as doping level and epitaxial layers width). In the photoluminescence spectra calculations three fitting parameters have been used. These parameters are GaAs/InGaAs valence band offset in strained quantum well, hole quasi Fermi level and inhomogeneous broadening. The PL peaks amplitudes and positions dependencies on the structure parameters were established. These dependencies can be used as the basis for pHEMT structure non-destructive diagnostics.

\section{Acknowledgement}

This work has been supported by the Ministry of Education and Science of Russia by the Agreement No 14.582.21.0010 dated 14 October 2015, unique project identity RFMEFI58215X0010 of Federal Target Program Research and development on priority directions of scientific and technological complex of Russia for 2014-2020. 